\title{
VIRTUAL PROTOTYPING: RESULTS ILLUSTRATE UTILITY IN DEVELOPING WEAPON SYSTEM REQUIREMENTS
}

\author{
Phillip J. Brown \\ Loral Vought Systems \\ P. O Box 650003 M/S MM-90 \\ Dallas, TX 75265 \\ 214-603-1258 \\ brown_pj@lvs-emh.lvs.loral.com
}

\author{
Jack K. Lavender, Jr. \\ Loral Vought Systems \\ P. O Box 650003 M/S MM-91 \\ Dallas, TX 75265 \\ 214-603-9339 \\ lavender@lvs-emh.lvs.loral.com
}

\section{Copyright (C) 1995 By Loral Vought Systems Corporation}

\begin{abstract}
The Department of Defense, in response to excruciating budget pressures, recurring system integration problems, and the continuing remarkable growth in computer capability and high resolution graphics, has seized on the promise of advanced distributed simulation technologies (including virtual prototyping) as a means for improving efficiency in developing integrated defense system products. From the perspective of a weapon systems contractor, benefits of virtual prototyping include: (a) tighter coupling of operational requirements with developer implementation concepts, (b) introduction of a rapidly reconfigurable tool for refining system requirements early in the systems development cycle, and (c) a means for improving the visibility of the system requirements analysis process. Examples of requirements changes identified as a result of virtual prototyping activity are from Loral Vought Systems experience with virtual prototyping on the Line-of-Sight AntiTank (LOSAT) weapon system program. Also included is a vision of where these tools, in the context of a weapon system, can be used to abet the system requirements development process.
\end{abstract}

\section{Introduction}

System Engineering, the technical discipline fostered by the Department of Defense (DoD) to deal with system complexity, is on the threshold of acquiring a computer-age tool enabling the profession to move closer to the trial-and-error roots fundamental to all of engineering. The tool, virtual prototyping, has already seen limited use in simulating weapon system performance on computer-generated battlefields. The DoD defines a virtual prototype as: a computer based simulation of a system or subsystem with a degree of functional realism comparable to a physical prototype (Garcia 1994). Early virtual prototypes have ranged from relatively simple computer models to full-scale man-in-the-loop weapon system mock-ups in which battlefield sounds and visual stimuli are piped into the operator's battle station.

The value of trial and error in developing products is well known in the defense industry. Years of working at the cutting edge of technology have resulted in contractors depending on a multi-layered sequence of testing to ferret out design shortfalls and design mistakes. Testing begins with individual parts, transitions to testing collections of parts (integration tests), and culminates in subjecting the assembled product to experiments in anticipated operational environments. The success of this verification approach, or process, should come as no surprise. Testing is an established engineering technique for advancing the state of the art, i.e., advances are the result of learning from failures (Petroski 1992).

\section{The Requirement Development Challenge}

Many program and project managers neither accept nor recognize the value of a systematic requirements development process. The result, in many instances, is a "paperwork sideshow" (Grady 1993). On the other hand, too many system engineering proponents appear overly eager to prescribe how companies should do system requirements analysis. What these proponents fail to acknowledge is that, in many instances, they are trying to force-fit an alien discipline into cultures that have evolved as a result of years of experience in successfully developing products. The challenge system engineering practitioners face is to merge the system requirements development process into existing product development cultures.

Relentlessly driving the urgent need for process improvement is the increasing use of system intelligence to control system functionality. Questions of 
information flow, control parameters, and responses must be decided early as a part of the system architecting activity (Rechtin 1991). The even harder task of specifying, designing, and testing the conceptual construct follows (Brooks 1987). In working these requirement issues, a problem we have experienced is providing a readily understood frame of reference for promoting productive dialogue among software, hardware, test and other affected domain representatives. Our solution has been extensive use of implementation examples to help resolve requirement definition and allocation questions.

Prototyping provides a rich vein of implementation examples. Abstract-appearing requirement issues suddenly become real in the context of relevant implementation experience. "Bottoms-up" product designers are brought face-to-face with the impact of their decisions on other system elements. Domain experts have a common frame of reference.

Engineering practitioners have relied for years on using hardware, and more recently software, prototyping to flush out design flaws and shortcomings. Prototypes bridge the gap between engineering design and the complexity of reality (Adams 1991). Now, with the technology for supporting virtual prototyping racing ahead (Pimentel 1993), it is time for the product development community to recognize, and embrace, virtual prototypes as cost efficient tools for resolving fundamental system requirements and implementation issues.

\section{Department of Defense Initiatives}

In 1992, at the behest of the Director of Defense Research and Engineering (DDR\&E), a task force on simulation, readiness, and prototyping was chartered to evaluate advanced simulation technology and to recommend initiatives to mature the technology and its applications (Defense Science Board 1993). At the conclusion of the study, the task force observed that the technology exists. Moreover, with appropriate help from the DoD, it could lead to revolutionary improvements in the way DoD does business.

Five recommendations were made. The first was to promote interoperability within both the DoD and commercial environments. The second was for the Joint Chiefs of Staff to join with DDR\&E to establish a constantly available advanced simulation joint warfare environment. Recommendation three was for the military to gain experience with the technology. Experience is to be garnered by conducting experiments and demonstrations. The fourth recommendation identified several tools and technologies needing DoD seed money. The final recommendation was to modify the DoD acquisition process to enable Modeling and Simulation $(M \& S)$ applications to take advantage of the ongoing rapid technology turnover of commercial products.

Results were immediate. A Memorandum of Agreement for the Joint Chiefs and DDR\&E to work together was signed in August 1992. Modeling and Simulation issues were identified and solutions suggested (Schoen 1993). The Defense Systems Management College published results of a DoD sponsored research effort to identify and assess the current uses of virtual prototypes (Garcia 1994). Work began at once and continues to this day as the Services strive to incorporate advanced simulation technology into a number of planned demonstrations and experiments.

The LOSAT program has been on the forefront of supporting DoD advanced simulation initiatives. A LOSAT virtual prototype was first employed in December 1990 in the SIMulation NETwork (SIMNET) developmental facility at Ft. Knox, Kentucky. The LOSAT virtual prototype was recognized, in a 21 May 1992 presentation to the Senate Armed Services Committee, for its contribution in a warfighting exercise. In that exercise, combat elements located in various parts of the country ambushed a column of T-72 tanks. Less well known, but central to this paper, is how we have used LOSAT virtual prototypes to resolve system requirements issues.

\section{LOSAT}

LOSAT is a highly mobile, all-weather, day/night, direct-fire anti-armor weapon system capable of defeating threat forces at a range of several kilometers. A sophisticated fire control system achieves hit-to-kill accuracies with a hypervelocity missile. Targets are defeated upon impact by a kinetic-energy kill mechanism. The LOSAT system is hosted on the Armored Gun System (AGS) platform developed by United Defense, LP. It is being developed for deployment with U.S. ground forces on a worldwide basis. Figure 1 shows the LOSAT on an AGS platform. Predecessor concepts used the Bradley Fighting Vehicle as the host vehicle.

The essential elements of LOSAT's Fire Control System (FCS) are a television sensor, a Forward Looking InfraRed (FLIR) sensor, a laser ranging and uplink device, several computers, and the controls and displays used by the soldier to control the system. The missile includes an on-board guidance processor that controls the flight trajectory to the target. During flight, guidance updates are sent via uplink laser to refresh missile knowledge of its position with respect to the target. These guidance updates are received by an aftlooking receiver. 


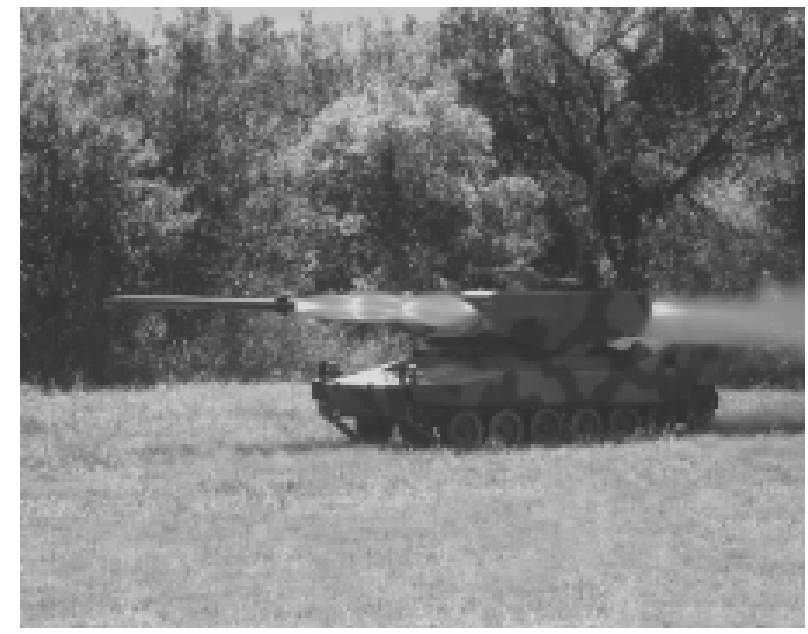

Figure 1. LOSAT on an AGS platform

Figure 2 depicts the target acquisition and engagement sequence. An engagement sequence begins by designating a target to be tracked. After track is established, the FCS computes the azimuth and elevation angles for the missile launcher and sends the missile's guidance processor launch information about the missile and the target. Examples of this information include: launch platform attitude, propellant temperature, wind speed, and wind direction. Upon launch the missile begins flying a predetermined trajectory to the target under the direction of the guidance processor. However, there are errors in guidance processor position and velocity calculations due to in-flight conditions which are impossible to predict. To correct for these errors, the fire control system updates the missile 30 times a second via the laser uplink. Each update tells the missile where it is relative to the target. The guidance processor recomputes the inertial guidance solution and commands the missile to make appropriate trajectory adjustments. Trajectory adjustments are made by firing attitude impulse control motors.

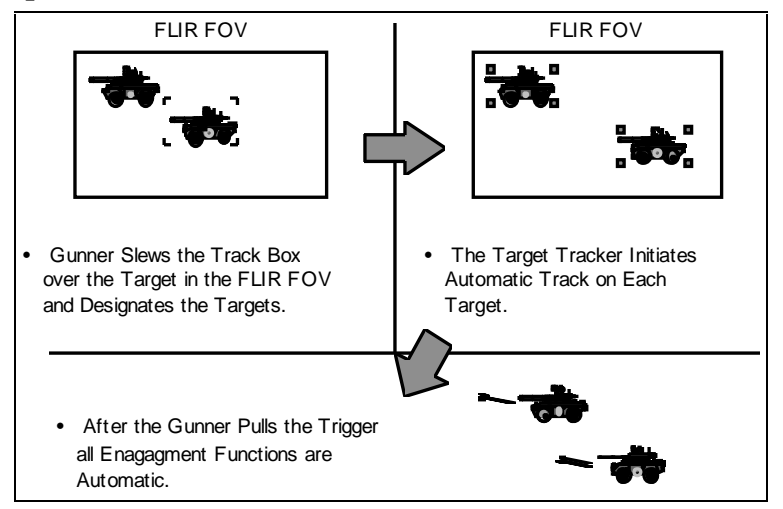

Figure 2. Target Acquisition, Tracking and Engagement
Maintaining target engagement timelines mandates a rapid-fire sequence of system actions. Accomplishing these tasks raised numerous questions about allocating functionality between man and machine. The use of virtual prototypes has proven invaluable in resolving task allocation issues.

\section{SIMNET}

SIMNET began in 1983 as a joint DARPA/Army project. It was to be a proof-of-principle technology demonstration of real-time, soldier-in-the-loop, battle engagement simulation and wargaming tool. Originally intended for military collective training, it soon became apparent that it could play a larger role in evaluating weapon system concepts prior to acquisition decisions (McDonough 1992).

LOSAT's SIMNET testbed simulates the LOSAT integrated FCS on a Bradley chassis. Figure 3 shows the LOSAT SIMNET simulator in use. The testbed was fabricated by BBN Corp. and Perceptronics Inc., the Army SIMNET contractors. It consists of a crew compartment in which the commander and gunner are seated side by side. The commander has simulated vision blocks, displaying a computer generated image visual system. Both the commander and gunner have simulated FCS controls and displays including control panels between actual LOSAT handgrips. There is a separate driver's station beside the commander's and gunner's crew compartments. Sound effects are simulated, and voice communication is via helmet headsets.



Figure 3. LOSAT SIMNET Simulator

Targets are generated from another workstation linked to the battlespace database. An operator, representing the commander of the opposing forces, calls up preprogrammed scenarios. Scenarios contain doctrinally correct numbers and types of targets and target velocities. Some targets are programmed to maneuver as attacking forces, others as allies. There are single targets, multiple targets, defensive scenarios, and offensive scenarios. 
Soldiers from Ft. Knox and Ft. Benning fought the LOSAT against these virtual forces. Fighting LOSAT included positioning the vehicle in an overwatch position, searching for and identifying targets, establishing track on highest priority targets, launching missiles at designated targets, and reloading missiles. Tests were conducted between December 1990 and April 1992. Problems with the initial configuration included completing multiple target "lock-on" before the first target tracked exited the field of view; having single targets at short range moving across the Field Of View (FOV) before lock-on could be achieved; subjects searching the wrong sectors for targets; and operators failing to notice when missile reload was needed (Davis 1992).

A number of functional allocation changes resolved these problems. One of the primary changes was to relocate control functions to facilitate time-critical tasks. At the soldiers' recommendation, the following requirement evolved: all controls that must be actuated while concentrating on the primary display shall be on the handgrips. This is an adaptation of the aircraft hands-on-throttle-and-stick (HOTAS) concept, eliminating the need to look away and visually find time-critical controls. Meeting this requirement resulted in a shortened timeline, reduced operator frustration, and fewer operator errors.

Another significant change involved FCS moding. Search had been conducted in a sight-slew mode in which either the FLIR or TV sensor was slewed to search the battlefield. The displayed scene had a crosshair at the sensor Line Of Sight (LOS). When a target was detected, the soldier selected the track-box mode, freezing the sensor LOS. The crosshair was then replaced with a track-box. The track-box was slewable within the fixed sensor FOV. Upon sizing the track box and placing it over the target, the soldier designated the target, commanding the FCS to start target track within the fixed scene. He then selected auto track, commanding the FCS to slew the sensor to align the target (or centroid of targets) with the sensor LOS.

SIMNET trials exposed a frequent inability to complete this sequence before moving targets left the FOV. This meant the soldier would have to select sightslew, break track on the target(s), and restart the sequence.

After an iterative process of considering various potential improvements, FCS modes were combined and automated. The track-box mode was deleted and the crosshair was replaced with the track box in sight-slew, resulting in the first target being in the center of the FOV when designated. Soldier selection of auto track was eliminated by making it automatic with target designate. The track box then could be slewed across the target tracking scene to designate other targets. This moding was refined subsequently to treat the track box as a target in track when establishing line-of-sight target-centroid tracking, thereby allowing an easy and natural means of searching for targets just outside the immediate FOV.

Two time-saving functions, automatic sensor scan and target cueing, were implemented and tested in SIMNET. Automatic scan eliminated the tedious task of manually slewing the sensor to retrace a search path. Automatic scan and cueing are used when LOSAT is emplaced for extended periods of time.

To attract crew attention to the need to reload or depart for resupply at the appropriate time, flashing missile count symbology was implemented in the LOSAT virtual prototype. As with all changes, testing verified improved system performance.

\section{HEDS}

SIMNET successes, in conjunction with DoD advanced distributed simulation initiatives, led to the development in 1993 of the LOSAT Human Engineering Development Station (HEDS). Figure 4 shows the HEDS simulator in use. The HEDS prototype was created to further improve the tactical system manmachine interface for the gunner's crew station. It was designed around a rapidly reconfigurable architecture.

The Distributed Interactive Simulation (DIS) standard is used to link HEDS to geographically dispersed simulations. DIS is based on IEEE standard 1278. DIS enables simulations of various types and levels of detail to play on a common virtual battlefield. DIS uses currently available commercial communications hardware.

HEDS can be linked into either the DIS net or SIMNET. Connectivity to DIS, and backwards compatibility with SIMNET, is accomplished with a Loral Advanced Distributed Simulation product named SNIP. SNIP (Simulation Network Interface Package) manages tasks required of the DIS node. Examples of tasks are coordinate system conversion, Ethernet communications, and keeping tabs on entity locations and battlefield events.

Opposing and allied forces interact with the HEDS via DIS. HEDS' ability to link into DIS was first demonstrated in a link to a constructive simulation of a Red Force attempting to breach Blue defenses. The LOSAT system was the only virtual prototype used in the battle.

The first use of HEDS was the development of functionality and menus for the programmable control panel. Improved FCS functionality resulted from repeatedly exercising the target acquisition and engagement procedure. Documenting these results produced further insights and improvements to the FCS. Three examples are provided below. 


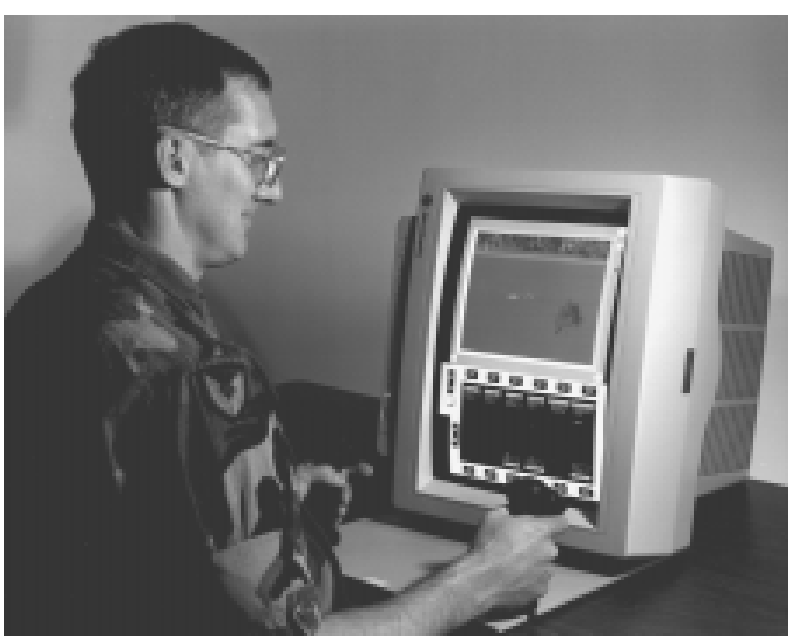

Figure 4. LOSAT HEDS Display

As described in the section on SIMNET, target cueing was developed as a feature to be used in combination with autoscan. A time-consuming initialization process was required to prepare for lengthy emplacements. This process was operationally acceptable in SIMNET warfighting exercises because target cueing was used only after a visual search determined there were no threat vehicles in the immediate area. While exercising manual designation of multiple targets in HEDS, it was apparent that a capability to utilize limited target cueing without entering the initialization mode could provide a significant reduction in the time required to attack enemy targets. This translates into improved survivability and higher probability of defeating clusters of targets.

When the soldier first selected target cueing, he was prompted with the message "Enter Ranges". The FCS functionality was modified to allow the target cuer to use laser rangefinder information without being in the initialization mode. When targets are detected, the soldier selects target cueing, slews the track box to a point above the targets, and ranges with the laser. The soldier then slews the track box to a point below the targets and ranges with the laser. This allows target cueing to be active in two fields of view. By implementing the target step feature with automatic track box sizing, the requirement to slew and size the track box over each target was eliminated.

Another enhancement further improved engagement functionality. At trigger pull, targets in autotrack go into "coast" mode. Coast is the resulting FCS tracker state when the system is unable to maintain active autotracking. It results in the track gates (tracker position symbol) maintaining the last known target velocity. The condition is displayed as a flashing track gate. If the target changes velocity during the coast period, the target and the track gate will diverge. The target tracker may be unable to reestablish auto track. To improve this, the FCS was modified to allow the operator to slew the track gate over the target. This increases the chances of reacquiring the target. If track is not re-established, the soldier is provided with a manual aim capability.

In order to upgrade the HEDS representation of the multiple missile environment, target-impact fireball effects were introduced on the operator's screen after impact of the first target. This fireball, or thermal cloud, has been observed in tests and is important because it can obscure subsequent targets and/or cause a missile tracker to lose track in mid-flight. Fireball interference in some situations resulted in engagement abort. With this added complexity, it became necessary to automatically calculate a target engagement sequence to minimize the potential negative effects of the fireball on the missile and target trackers.

The requirements issue was how to keep the soldiers in the decision-making process. User representatives decided the system should: (1) display the sequence and engagement hold (not abort) situations and (2) give the soldier an override capability. The system now calculates the size and anticipated position of the fireball and displays new symbology representing (a) the fireball, (b) the crosswind direction, and (c) the target engagement sequence. An optimal engagement sequence is determined by the system. New symbology was added to indicate any targets in hold. This implementation has been tested and deemed operationally sound.

Early resolution of these and other functional allocation issues substantiates the contention that virtual prototypes add value to the requirements development process.

\section{FUTURE DIRECTIONS}

Effective communication between man and machine has been a central concern of the aerospace/defense industry for 30-plus years (Fogel 1963). The birth and phenomenal growth of the personal computer industry has created an explosion of new research and ideas for improving human/computer interfaces (Laurel 1990). The common thread in this work is how to extract maximum advantage from intelligent machine advances while maintaining human control.

Determining the proper functional allocation between man and machine is complicated by man's limited sensory capacities and relatively slow information processing speeds. For instance, vision is a human's primary information gathering channel. This modality, if one accepts the provocative hypothesis of the executive director of research at AT\&T's Bell Labs (Lucky 1991), has an information processing limit of 50 
bits per second. Experimentation is the quickest way for verifying solutions for overcoming human sensory limitations and effectively using poorly understood cognitive processes. Virtual prototyping will, over time, see increasing use in resolving difficult man-machine functional allocation questions.

Economics will eventually persuade decision makers of the merits of more extensive use of virtual prototypes. Producing a physical prototype with tactical capabilities can take years and hundreds of millions of dollars. It has low training throughput and its operation may be dangerous. Shortcomings discovered late in the system testing process are often difficult and costly to correct.

The LOSAT program has developed and delivered working virtual prototypes in less than a year. Costs were orders of magnitude less than those for developing a tactical physical prototype. Soldiers have attested to the verisimilitude of the LOSAT virtual prototypes. The series of interactive experiments conducted over the past four years has given us confidence that the tool is a viable means for improving system functionality.

LOSAT will continue to use virtual prototypes to aid in resolving key interface issues, such as vehicle command and control, threat detection, and countermeasures. Prototype trials also will assist in establishing crew requirements for system checkout, maintenance, and embedded training.

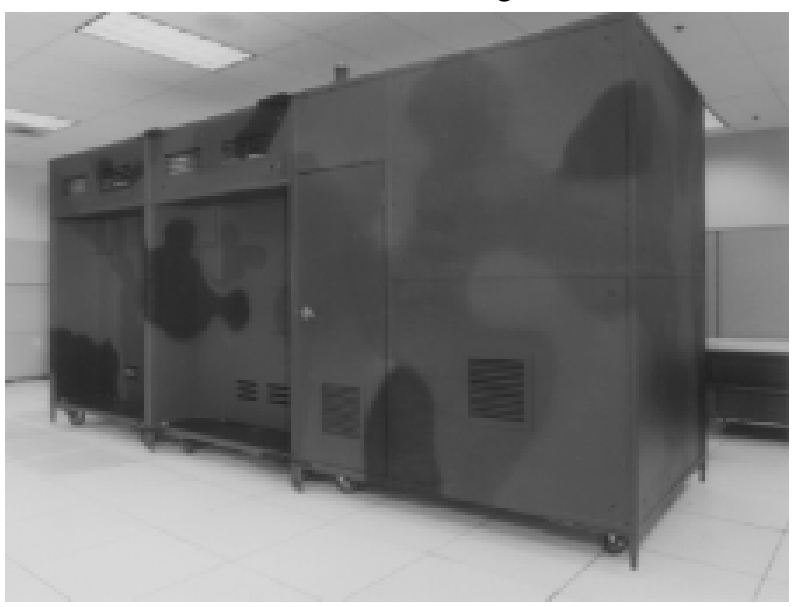

Figure 5. External view of DISCSS

Figures 5 and 6 picture the LOSAT Distributed Interactive Simulation Crew Station Simulator (DISCSS). DISCSS is the next generation LOSAT virtual prototype and will replace the existing LOSAT SIMNET device located at Ft. Knox. It is composed of a simulated LOSAT crew compartment and contains all crew station controls and displays. DISCSS is situated at Ft. Benning, Georgia in the dismounted battlelab facility. It will be used to extend our knowledge of how to maximize LOSAT utility on the battlefield.
It is important to know that initial plans for DISCSS had the LOSAT FCS hosted on a Bradley Fighting Vehicle. Concept implementation proceeded accordingly. When programmatic changes dictated hosting the FCS on an AGS chassis, a very modest investment in time and money produced the desired prototype changes. This, contrasted with the investment that would have been required to make comparable changes to a physical prototype, is an excellent example of why virtual prototypes are so attractive for concept definition work.

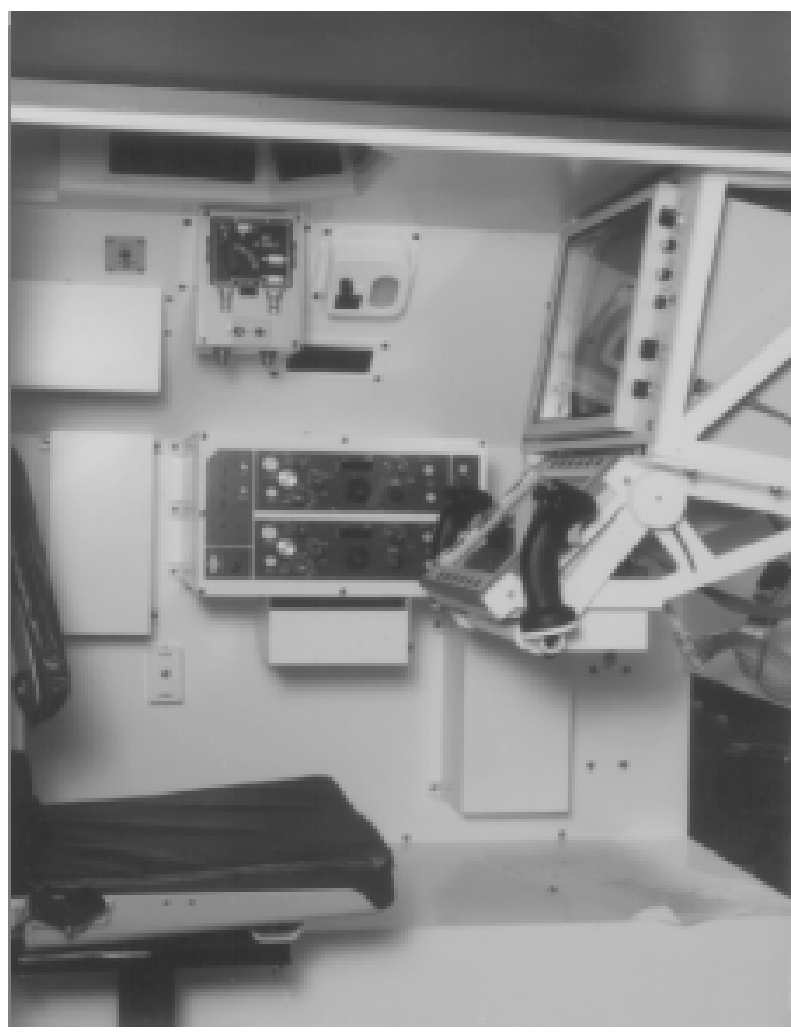

Figure 6. Internal view of DISCSS crew station

Tantalizing opportunities for interface improvement lie just over the horizon. Expert systems, fuzzy logic, adaptive filters, and neural networks are terms evoking the use of higher machine intelligence. Research in immersion technologies suggests consideration of headmounted displays, wired gloves, force balls, six-degreeof-freedom mice, and biologic input sensors. Some particularly intriguing work is being done with virtual reality information management systems to increase the amount of information people can meaningfully manage (Fairchild 1993).

Use of virtual prototypes early in the product development life cycle adds the needed dimension of operational constraints to the requirements allocation process. Early use also stimulates thoughts on how to exploit man's unique cognitive skills to achieve an optimal human-machine design balance. 


\section{SUMMARY}

System engineering practitioners need to find ways to integrate the requirements development process into existing organizational cultures. Experience with virtual prototypes has demonstrated their value in identifying and resolving man-machine functional allocation issues. Although the use of virtual prototypes will never replace traditional engineering activities, they do promise to dramatically reduce weapon system development costs.

\section{ACKNOWLEDGMENT}

Much of the credit for the accomplishments related above belongs to our partners in government. The LOSAT Project Office at Redstone Arsenal, Alabama identified the opportunities and provided the vision of the road ahead. Soldiers from Ft. Benning and Ft. Knox gave the needed positive and negative feedback. Project office personnel have been a continuing source of energy and expertise to get us over the inevitable hurdles.

\section{REFERENCES}

Adams, James L., Flying Buttresses, Entropy, and $O$ Rings, The World of the Engineer, Harvard University Press, Cambridge, Massachusetts, 1991.

Brooks, Frederick P., "No Silver Bullet, Essence and Accidents of Software Engineering." Computer 20, 4, 10-19, April 1987.

Davis, Gardner, "LOSAT SIMNET Simulation Concept Evaluation Program and System Design Phase Summary," LOSAT-SDP/92DIR-0276, 9 June 1992.

Defense Science Board, Impact of Advanced Distributed Simulation on Readiness, Training and Prototyping, Office of the Under Secretary of Defense for Acquisition, Washington, D.C., January, 1993.

Fairchild, Kim M., "Information Management Using Virtual Reality-Based Visualizations," Chapter 3 in Virtual Reality: Applications and Explorations, Academic Press Professional, Cambridge, Massachusetts, 1993.

Fogel, Lawrence J., Biotechnology: Concepts and Applications, Prentice-Hall, Inc., Englewood Cliffs, N.J., 1963

Garcia, Albert B., Grocke, Robert P., and Johnson, Nelson P., Virtual Prototyping: Concept to Production, Defense Systems Management College Press, Ft. Belvoir, Virginia, March, 1994.

Grady, Jeffrey O., "In Defense of the Traditional System Requirements Analysis Process", Proceedings of the Third Annual International Symposium, National Council on Systems Engineering (NCOSE), Arlington, Virginia, July 26-28, 1993.
Laurel, Brenda (editor), The Art of Human-Computer Interface Design, Addison-Wesley Publishing Company, Menlo Park, California, 1990.

Lucky, Robert W., Silicon Dreams: Information, Man, and Machine, St. Martin's Press, New York, 1991.

McDonough, James, "Doorways to the Virtual Battlefield," in Proceedings of the 1992 Virtual Reality Conference, Carnegie-Mellon University, Pittsburg, Pennsylvania, 1992.

Petroski, Henry, To Engineer is Human: The Role of Failure in Successful Design, Vintage Books, New York, 1992.

Pimentel, Ken, and Teixera, Kevin, Virtual Reality: Through The New Looking Glass, Intel/Windcrest/McGraw-Hill, Inc., New York, 1993.

Rechtin, Eberhardt, Systems Architecting: Creating and Building Complex Systems, Prentice-Hall, Inc., Englewood Cliffs, New Jersey, 1991.

Schoen, Joel M., and Starr, Stuart H., "Issues Driving the Effective Use of Modeling \& Simulation (M\&S) in the Acquisition Process," Proceedings of the Summer Computer Simulation Conference, Boston, Massachusetts, 19-21 July 1993.

\section{BIOGRAPHIES}

Phillip J. Brown is a Technical Project Manager, LOSAT System Engineering, for Loral Vought Systems. $\mathrm{He}$ has a Bachelor of Civil Engineering from the Georgia Institute of Technology and a Master of Science in Industrial and System Engineering from the Ohio State University. His 28 years experience include system engineering work on aircraft, cruise missiles, direct fire rockets, air defense missiles, and satellite systems. He is a registered professional engineer, a member of the National Society of Professional Engineers, and a member of NCOSE.

Jack K. Lavender, Jr. is an Operations Analyst for Loral Vought Systems. He has a Bachelor of Arts in Mathematics and a Master of Arts in Mathematics from the University of Texas at Arlington. His 13 years of experience include Master Dimensions work on aircraft, scientific computing support for advanced surface-to-air missile systems, and research of advanced computing techniques for non-cooperative target recognition. He is currently leading the system engineering virtual prototyping implementation effort for LOSAT. He teaches $\mathrm{C}$ and $\mathrm{C}++$ programming courses through Dallas County Community College District. 